\title{
Statistical Goodness Factor ' $\Gamma$ ' for Image Fusion Algorithm Based on UGGD Parameters
}

\author{
MSA Srivatsava, T. Ramashri, K Soundararajan
}

\begin{abstract}
In this paper we propose a novel pyramid decomposition based Image fusion metric, Gamma Factor or Goodness of Fit ' $T$ ' which describes the statistically amount of information fused by the image fusion algorithm. We first apply steerable pyramid decomposition and then a fitting model for Univariate Generalised Gaussian Distribution (UGGD) parameter estimation. From the UGGD; $P$ and $S$ fitting model coefficients are computed. To estimate the optimum weights for computation a huge data set of complimentary images are used. Using these weights, amount of information contributed by each image to form a fused image can be estimated. Experimental results show the tremendous matching with the quantise information
\end{abstract} Image

Keywords: Pyramid, UGGD, Weights, fitting model, Fused

\section{INTRODUCTION}

THE objective of image fusion is to coordinate data from different info images to make a combined one that is progressively instructive for human or machine observation as contrasted with any of the information images [1]. Image fusion procedures have been utilized in different application zones counting remote detecting, biomedical imaging, and multi-exposure multi-center image coordination[2]. In optical remote detecting, a gathering of sensors may cooperate, each of which catches some particular parts of ghastly as well as spatial data[3]. Melding both the spatial and otherworldly data from all sensors gives an increasingly enlightening image, and as it were the melded image should be put away for resulting investigation of the scene [4]. In biomedical imaging, diverse imaging modalities are corresponding in nature in securing unique parts of organic structures and exercises. For instance, attractive reverberation imaging (MRI) is frequently helpful in uncovering anatomical structures though metabolic exercises might be caught all the more dependably utilizing positron discharge tomography (PET). By utilizing fusion innovations, it is conceivable to get a single image that adequately portrays anatomical structures also, metabolic exercises at the same time [5]. Because of the vast number of uses and the decent variety of fusion procedures, impressive endeavours have been made to create target execution measures for image fusion.

Revised Manuscript Received on February 05, 2020.

* Correspondence Author

MSA Srivatsava, Head and Director, Department of ECE, JNTUA College of Engineering, JNTU Anantapur, Anantapuramu.

Prof. Dr.T.Ramashri, Head, Department of ECE, JNTU College of Engineering, JNTU Anantapur, Anantapuramu.

Prof.Dr.K.Soundararajan, Professor, Department of ECE, JNTU College of Engineering, JNTU Anantapur, Anantapuramu.

(C) The Authors. Published by Blue Eyes Intelligence Engineering and Sciences Publication (BEIESP). This is an open access article under the CC BY-NC-ND license (http://creativecommons.org/licenses/by-nc-nd/4.0/)
Customarily, the appraisal of a fusion plot is conveyed out by abstract assessment, which is known to be moderate, costly, and above all, can't be installed into robotized structures for framework and parameter enhancements. A significant option in contrast to abstract assessment is objective image fusion estimates that are steady with human visual system. There are many methods proposed by researchers, like Mutual Information [6], relative amount of edge information preserved [7], measures based on SSIM[8], some of them were based on Contrast Visual System based on Human Visual System[9], Local amplitude, Contrast Preservation leads to Fusion Quality Measure[10]. Natural image wavelet coefficients have zero high peaks with tails being wider [11]; we adopt a natural scene statistics based model using UGGD [12].

\section{SYNGO FASTVIEW SYSTEM}

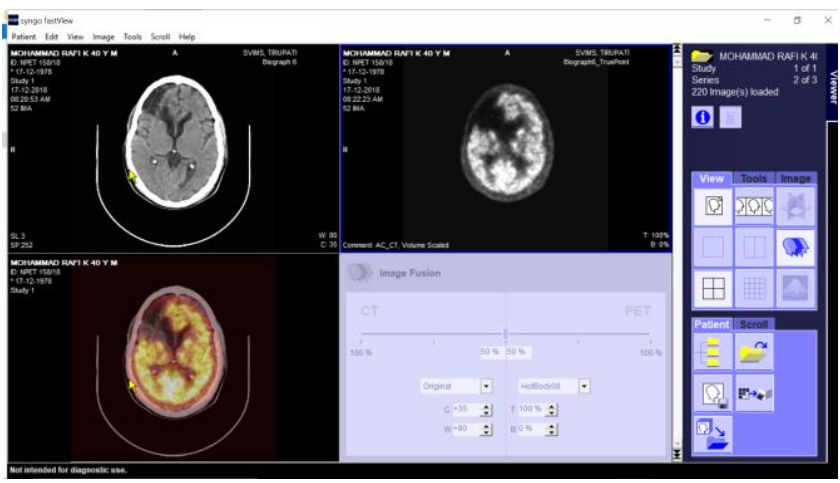

\section{PROPOSED METHOD}

Formulae used:

Generalised Gaussian Distribution:

A random variable $\mathrm{X}$ is distributed as generalised Gaussian if its probability distribution function ( $p d f)$ is given by

$$
g g(x ; \mu, \sigma, p)=\frac{1}{2 \Gamma\left(1+\frac{1}{\mathrm{p}}\right) \mathrm{A}(\mathrm{p}, \mathrm{s})} \mathrm{e}^{-}\left|\frac{\mathrm{x}-\mu}{\mathrm{A}(\mathrm{p}, \sigma)}\right|^{\mathrm{p}}, \mathrm{x} \in \mathrm{R}
$$

Where $\mu \in \mathrm{R}, \mathrm{p}, \sigma>0$ y $\mathrm{A}(\mathrm{p}, \sigma)=\left[\frac{\sigma^{2} \mathrm{r}\left(\frac{1}{\mathrm{p}}\right)}{\mathrm{r}\left(\frac{3}{\mathrm{p}}\right)}\right]^{1 / 2}$

The parameter $\mu$ is the mean $\mathrm{A}(\mathrm{p}, \sigma)$ is a scaling factor which allows $\operatorname{Var}(\mathrm{x})=\sigma^{2}$ and $\mathrm{p}$ is the shape parameter[12].

\section{Weights Computation:}

$\alpha(\mathrm{MRI})+(1-\alpha) \mathrm{PET}=$ Fusion

$\mathrm{W}_{\mathrm{p}}\left(\mathrm{P}_{\mathrm{m}}\right)+\left(1-\mathrm{W}_{\mathrm{p}}\right)\left(\mathrm{P}_{\mathrm{p}}\right)=\mathrm{P}_{\mathrm{f}}$

$\mathrm{W}_{\mathrm{s}}\left(\mathrm{S}_{\mathrm{m}}\right)+\left(1-\mathrm{W}_{\mathrm{s}}\right)\left(\mathrm{S}_{\mathrm{p}}\right)=\mathrm{S}_{\mathrm{f}}$

$\left[\mathrm{W}_{\mathrm{p}}, \mathrm{W}_{\mathrm{s}}\right]$ - Optimum weights

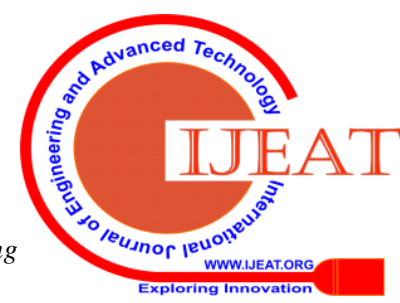




\section{Statistical Goodness Factor ' $\Gamma$ ' for Image Fusion Algorithm Based on UGGD Parameters}

$$
\begin{aligned}
& \mathrm{W}_{\mathrm{p}}=\left[\left(\mathrm{P}_{\mathrm{f}}-\mathrm{P}_{\mathrm{p}}\right) /\left(\mathrm{P}_{\mathrm{m}}-\mathrm{P}_{\mathrm{p}}\right)\right] \\
& \mathrm{W}_{\mathrm{s}}=\left[\left(\mathrm{S}_{\mathrm{f}}-\mathrm{S}_{\mathrm{p}}\right) /\left(\mathrm{S}_{\mathrm{m}}-\mathrm{S}_{\mathrm{p}}\right)\right]
\end{aligned}
$$

\section{Proposed Method:}

Flow chart

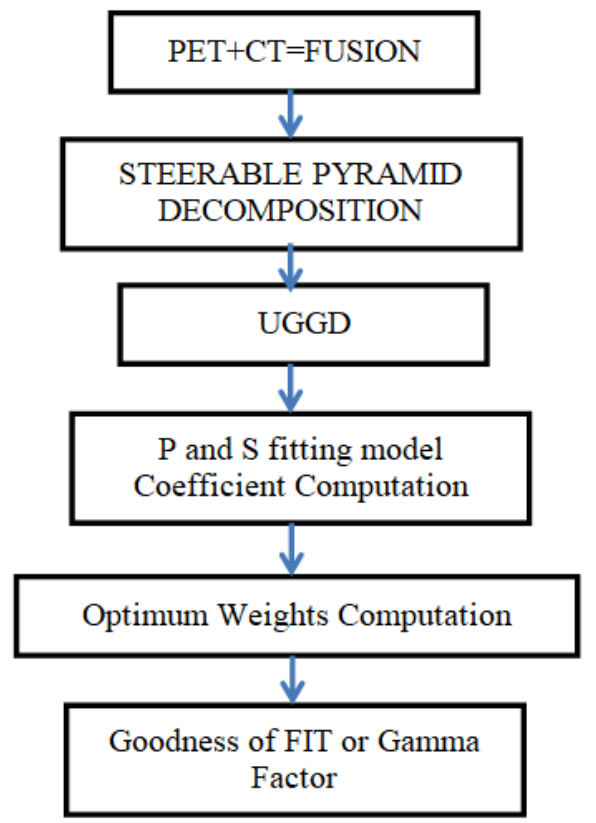

Fig1: Block diagram of our proposed method

Our Algorithm can be explained in steps as follows Step 1: 50 images of each CT( $\left(\mathrm{I}_{1}\right)$, PET $\left(\mathrm{I}_{2}\right)$ and Fused images $\left(\mathrm{I}_{3}\right)$ are taken from 'Siemens Syngo Fast View system' Step 2: Steerable Pyramid Decomposition is applied on these 3 sets of images $I_{1}, I_{2}$ and $I_{3}$.

Step 3: Fitting model using Univariate Gaussian Distribution is applied

Step 4: P and S fitting model coefficients are computed for the three sets of images $\mathrm{I}_{1}, \mathrm{I}_{2}$ and $\mathrm{I}_{3}$.

Step 4: Optimised Weights are computed from the Mode of $\mathrm{P}$ and $\mathrm{S}$ train as stated in equation $2 \mathrm{a} \& \mathrm{~b}$

Step 5: Goodness of fit implies the information of image information ratio.

Resur
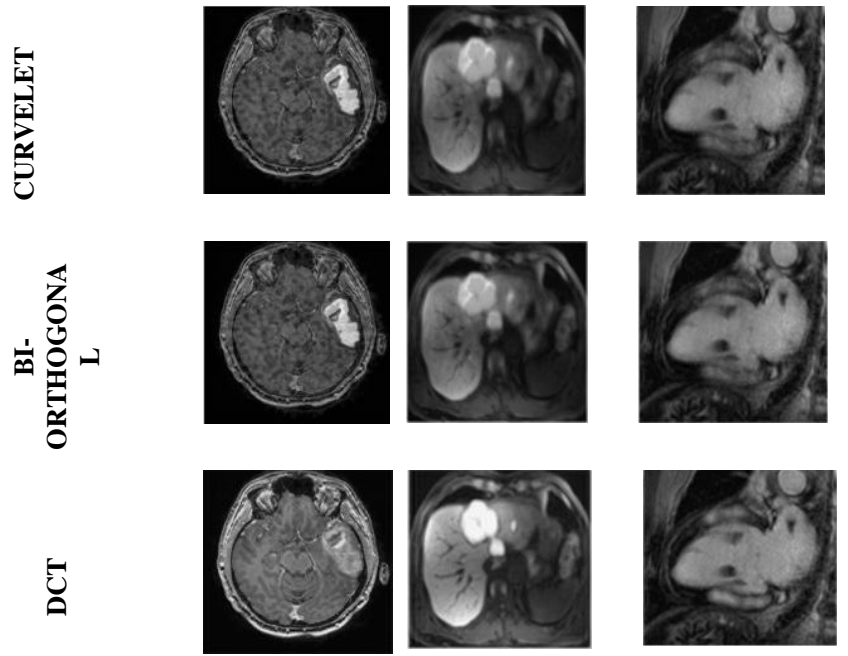
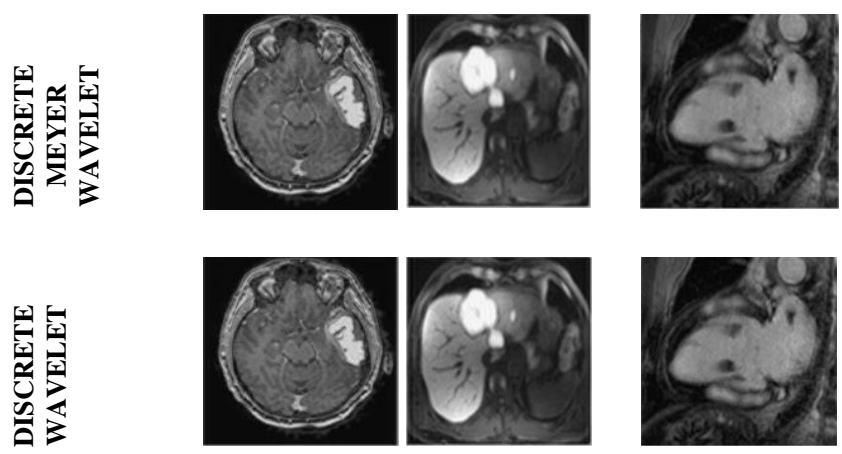

Table

\begin{tabular}{|c|c|c|c|c|c|}
\hline & $\begin{array}{c}\text { \% of } \\
\text { Image } \mathrm{I}_{1}\end{array}$ & $\begin{array}{c}\text { \% of Image } \\
\mathrm{I}_{2}\end{array}$ & $\mathrm{~W}_{\text {pOPT }}$ & $\mathrm{W}_{\text {sOPT }}$ & $\begin{array}{c}\text { Goodness of } \\
\text { fit }\end{array}$ \\
\hline 1 & 50 & 50 & 0.2589 & 0.0129 & 0.3242 \\
\hline 2 & 10 & 90 & 1.0236 & 1.0289 & 0.8171 \\
\hline 3 & 90 & 10 & 1.0259 & 1.0296 & 0.1969 \\
\hline
\end{tabular}

\begin{tabular}{|c|c|}
\hline WP SET & WS SET \\
\hline 0.258919721169276 & 0.0129039219227337 \\
\hline 0.920869667596737 & 0.484466087733380 \\
\hline 0.698261871824475 & 0.346120718626884 \\
\hline 1.00216385936604 & 0.541660355837595 \\
\hline 0.348707953102714 & 0.270408825372705 \\
\hline 3.18125488971714 & 0.707086191914614 \\
\hline 0.550324548185878 & 0.288144403695189 \\
\hline 0.810601709837845 & 0.570757133804984 \\
\hline 2.27445747213095 & 0.370691172877168 \\
\hline 10.2838869879386 & 0.726758487332387 \\
\hline 1.57054363843352 & 0.769382809562805 \\
\hline 0.656061965439615 & 0.734637210513457 \\
\hline 0.910287766840676 & 1.16283914319346 \\
\hline 1.02836516343699 & 1.20655897847161 \\
\hline 1.50042407867641 & 0.218076596379786 \\
\hline 1.54026494468142 & 0.148255356190310 \\
\hline 1.13709042942767 & 1.74793603312615 \\
\hline 2.95473576684454 & 0.206940414343039 \\
\hline 1.17240434280698 & 2.61060227419103 \\
\hline 0.863785254593527 & 0.508054458050773 \\
\hline 1.04672408245578 & 1.42257166001318 \\
\hline 0.990249305242783 & 1.07706032735647 \\
\hline 1.05977952302857 & 1.34709690495616 \\
\hline 1.00234889542323 & 1.03455894304822 \\
\hline 1.01429557036646 & 1.02264559643994 \\
\hline 1.04053575247810 & 1.16719053134328 \\
\hline 0.995587289624544 & 1.04662170761712 \\
\hline 1.03810574914396 & 1.13527802052763 \\
\hline 0.957770816261636 & 1.00539398311829 \\
\hline 1.06388240992019 & 1.08245613180302 \\
\hline 0.997205199105371 & 1.03424749981303 \\
\hline 1.02714348323974 & 1.08664045030756 \\
\hline 0.983999314741400 & 1.00721348837141 \\
\hline 1.02272195125715 & 1.06079623396757 \\
\hline 0.980959527866980 & 1.02994382110140 \\
\hline 0.951619330408008 & 1.00380140914825 \\
\hline 0.786689933621587 & 0.900993636743907 \\
\hline 1.21700152885210 & 493.204071071688 \\
\hline 0.994710951768829 & 1.93558803040113 \\
\hline 1.10275637982769 & 3.16411825775234 \\
\hline 1.33807910425866 & 0.274716218508962 \\
\hline
\end{tabular}

Table: Weighted sums of MRI and CT images.

\section{CONCLUSION}

The proposed method opens a new method of evaluating and creating a method of fusion by using UGGD

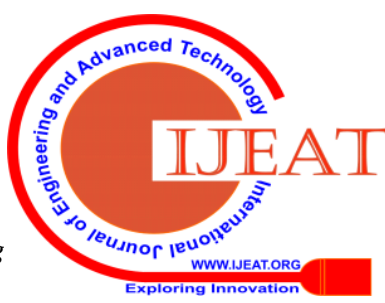


(Univariate Generalised Gaussian distribution) results clearly depict the trade-off between the image fitting factors based on the weighted sums $\mathrm{P}$ and $\mathrm{S}$. This new factor ' $\mathrm{r}$ 'will be a reference factor which can be considered as a Goodness of fit function.

\section{REFERENCES}

1. No-reference Stereoscopic Image Quality Assessment Using Natural Scene Statistics Balasubramanyam Appina n, Sameeulla Khan,Sumohana S.Channappayya , Signal Processing: Image Communication 43 (2016)1-14

2. Y. Hu, S. K. Mirzac, J. G. Jarvikb, P. J. Heagerty, and D. R. Haynor, "MR and CT image fusion of the cervical spine: a noninvasive alternative to CT-Myelography," in Proceedings of SPIE, vol. 5744. Bellingham, WA: SPIE, 2005

3. C. A. Karlo, I. Steurer-Dober, M. Leonardi, C. W. A. Pfirrmann,

4. M. Zanetti, and J. Hodler, "MR/CT image fusion of the spine after spondylodesis: a feasibility study,” European Spine Journal, vol. 19, pp. 1771-1775, 2010.

5. R. Rensink, "Change blindness," Annual Review of Psychology, vol. 53, pp. 245-277, 2002.

6. R. A. Deyo, S. K. Mirza, J. A. Turner, and B. I. Martin, "Overtreating Chronic Back Pain: Time to Back Off?" Jour Am Board Fam Med, vol. 22, no. 1, pp. 62-68, 2009.

7. P. L. Owens, M. Woeltje, and R. Mutter, "Emergency Department Visits and Inpatient Stays Related to Back Problems," 2008. [Online].

8. Available: http://www.hcup-us.ahrq.gov/reports/statbriefs/sb105.pdf

9. H. Li, B. S. Manjunath, and S. K. Mitra., "Multisensor Image Fusion using the Wavelet Transform," Graphical Models and Image Processing, vol. 57, no. 3, pp. 235-245, 1995.

10. J. Núńez, X. Otazu, O. Fors, A. Prades, V. Palà, and R. Arbiol, "Multiresolution-Based Image Fusion with Additive Wavelet Decompo- sition," IEEE Transactions On Geoscience And Remote Sensing, vol. 37, no. 3, pp. 1204-1211, May 1999.

11. L. Yang, B. L. Guo, and W. Ni, "Multimodality medical image fusion based on multiscale geometric analysis of contourlet transform," Neu-rocomputing, vol. 72, pp. 203-211, 2008.

12. G. K. Matsopoulos, S. Marshall, and J. N. H. Brunt, "Multiresolution morphological fusion of MR and CT images of the human brain," IEEE Proceedings on Vision Image Signal Processing, vol. 141, no. 3, pp. 137-142, 1994.

13. Natarajan P, N.Krishnan, Sugavanesh C, "Brain MRI Image Edge Detection Using Top-Hat Mathematical Morphology”, 2011 IEEE International Conference on Computational Intelligence and Computing Research, PP 120 - 123, Dec 2011.

14. Natarajan P, N.Krishnan, Rajesh N, "Brain MRI Image Edge Detection Using Gradient Method”, 2011 IEEE International Conference on Computational Intelligence and Computing Research, PP 124 - 126, Dec 2011.

\section{AUTHORS PROFILE}

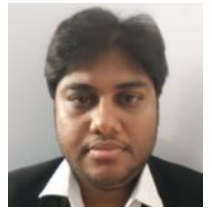

MSA Srivatsava, Completed his M.tech from SV University Tirupati, Currently Pursuing $\mathrm{PhD}$ in the department of ECE from JNTUA Anantapur and working as a Product Head and Director for Mindtronix (Robots manufacturing company). His research areas are Watermarking, Image fusion and Medical image applications.

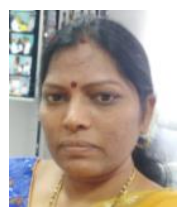

Prof.Dr.T.Ramashri, Currently working as Head of the department of ECE, Guided 10 PhD's till date. Published More than 125 National and International reputed journals and more than 90 National and International Conferences. Areas of interest are Image Processing, VLSI, Network Security, Computer Networks.

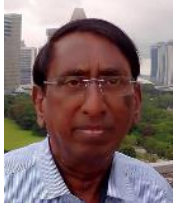

Prof.Dr.K.Soundararajan, A Retd professor of ECE from JNTU College of Engineering, JNTU Anantapur, Anantapuramu. His role was instrumental in developing the department and University as well, He also has served the university in various positions as Professor, HOD,Principal, OSD-VC and many more to add. He guided many $\mathrm{PhDs}$ and published more than Hundreds of National and International Publications and Conferences. His areas of Interests are Image Processing, VLSI, Embedded systems, Wireless communication and Microprocessors. 